1. E. P. Merkes \& W. T. Scott, "Continued fraction solutions of the Riccati equation," J. Math. Anal. Appl., v. 4, 1962, pp. 309-327. MR 25 \#4167.

2. W. FaIr, "Padé Approximations to the Solution of the Riccati Equation," Math. Comp., v. 18, 1964, pp. 627-634. MR 29 \#6630.

3. Y. L. Luke, "The Padé table and the r-method," J. Math. and Phys., v. 37, 1958, pp. 110-127. MR 20 \#5558.

4. H. T. DAvis, Introduction to Nonlinear Differential and Integral Equations, Chapter 8, Jover, New York, 1962.

5. W. E. Simon, "Numerical technique for solution and error estimate for the initial value problem," Math. Comp., v. 18, 1965, pp. 387-393.

6. H. S. WALL, Analytic Theory of Continued Fractions, Van Nostrand, New York, 1948. MR 10, 32. 25.

7. O. Perron, Die Lehre von den Kettenbrüchen, Vol. II, Teubner, Stuttgart, 1957. MR 19,

\title{
On the Evaluation of the Incomplete Gamma Function
}

\section{By Roy Takenaga}

0. Abstract. The accurate evaluation of the $\chi^{2}$ distribution for high degrees of freedom by the usual methods is very difficult (even with a digital computer) because the series to be evaluated would become unbearably long. Also, when a series becomes long, more precision in the numbers used is required in order to offset the effects of round-off errors. On a computer this would mean the use of multiple precision. Accurate tables can be, and have been, prepared by use of the Cornish-Fisher approximation. Comparison of the table values with the values obtained by the method in the writer's paper show that these tables have an accuracy of about six significant figures. For practical purposes there seems to be no lack of $\chi^{2}$ tables for high degrees of freedom. The method in the writer's paper is still useful in checking on the accuracy of tables computed by approximate methods or in producing tables with more significant figures. With single precision it can produce tables of seven figure accuracy at a speed far better than could be by the usual accurate methods. Some unique and useful tables can be produced using this method.

1. Introduction. The incomplete gamma function is defined by K. Pearson [2, p. v] to be

$$
\Gamma_{y}(p+1)=\int_{0}^{y} t^{p} e^{-t} d t, \quad-1<p, 0 \leqq y .
$$

This paper presents a method of evaluating the ratio

$$
F_{p}(y)=\Gamma_{y}(p+1) / \Gamma(p+1), \quad-1<p, 0 \leqq y,
$$

for cases in which $p$ is greater than about 1j. For the lower values of $p$ there are a number of integration methods. Among them is the formula [2, p. xv]

$$
\begin{aligned}
F_{p}(y)=\frac{e^{-y} \cdot y^{p+1}}{\Gamma(p+2)}\{1+ & \frac{y}{p+2}+\frac{y^{2}}{(p+2)(p+3)} \\
& \left.+\cdots+\frac{y^{k-1}}{(p+2)(p+3) \cdots(p+k)}+\cdots\right\} .
\end{aligned}
$$

Received January 24, 1966. 
When $p$ is large there are a number of difficulties in using this formula. For the usually desired values of $y$, such that $0.005 \leqq F_{p}(y) \leqq 0.995, e^{-y}$ becomes extremely small and $y^{p+1}$ becomes extremely large even if $y$ is restricted to be less than $p$ (there is a similar formula for large values of $y$ ). Of course, $\Gamma(p+1)$ becomes large, too, but the biggest difficulty is in obtaining the desired accuracies from the series, which tends to get long, causing serious round-off errors. On the IBM 7090 computer, 30 seems to be the top permissible value of $p$. After that value the forementioned troubles become serious.

Method $E^{\prime}$, on p. xviii of [2], describes a method of evaluating (2) when $p$ is high, called "the expansion in normal moment functions". Its utility was limited to extremely high values of $p$. Even for $p=100$, it integrated $F_{p}{ }^{\prime}(y)$ for only a small distance around the point $y=p$. Now in 1931 Wilson and Hilferty [3] showed, in effect, that for large $p$ the random variable represented by the cube root of $y$ had a distribution much closer to normality, as measured by the first four moments, than the distribution of $y$. This paper develops method $E^{\prime}$ for the distribution resulting from taking the cube root of $y$. The result has been programmed on the IBM 7094. It was found that this method will maintain six significant-figure accuracy for values of $y$ such that $0.005 \leqq F_{p}(y) \leqq 0.995$, even for $p$ as low as 14 . For higher accuracies the range must be cut down for the lower $p$ 's. Formula (3) and corresponding formulae for the other tail may be used to take over the tails.

\section{Transformation of the Integrand. Starting with}

$$
F_{p}^{\prime}(y)=\frac{y^{p}}{\Gamma(p+1)} e^{-y}
$$

let

$$
y=\left(\frac{x+a}{b}\right)^{3}
$$

where $a$ and $b$ are constants to be determined later (note that $y$ is monotonic increasing if $a>0$ and $b>0$ ). With $x$ as the new variable, the corresponding integrand (derived density function) is

$$
f(x)=\frac{3}{b}\left(\frac{x+a}{b}\right)^{3 p+2} \exp \left[-\left(\frac{x+a}{b}\right)^{3}\right] \cdot \frac{1}{\Gamma(p+1)} .
$$

Taking the log of expression (6),

$$
\log f(x)=\log \frac{3}{b}+(3 p+2) \log \left(\frac{x+a}{b}\right)-\left(\frac{x+a}{b}\right)^{3}-\log \Gamma(p+1),
$$

and using the Stirling asymptotic expansion for $\log \Gamma(p+1)$, this becomes

$$
\begin{aligned}
\log f(x)= & \log \frac{3}{b}+(3 p+2) \log \frac{a}{b}+(3 p+2) \log \left(1+\frac{x}{a}\right) \\
& -\left(\frac{a}{b}\right)^{3}\left[1+3 \frac{x}{a}+3\left(\frac{x}{a}\right)^{2}+\left(\frac{x}{a}\right)^{3}\right] \\
& -\left(p+\frac{1}{2}\right) \log p+p-\frac{1}{2} \log 2 \pi-\frac{1}{12 p}+\frac{1}{360 p^{3}}-\cdots
\end{aligned}
$$


Assuming $|x / a|$ to be less than one,

$$
\log \left(1+\frac{x}{a}\right)=\frac{x}{a}-\frac{1}{2}\left(\frac{x}{a}\right)^{2}+\frac{1}{3}\left(\frac{x}{a}\right)^{3}-\frac{1}{4}\left(\frac{x}{a}\right)^{4}+\cdots .
$$

Now we assume that $a$ and $b$ satisfy the equations

$$
(3 p+2)=3(a / b)^{3}
$$

and

$$
\frac{1}{2}(3 p+2)+3(a / b)^{3}=a^{2} / 2 .
$$

Solving for $a$ and $b$,

$$
a=3\left(p+\frac{2}{3}\right)^{1 / 2}
$$

and

$$
b=3\left(p+\frac{2}{3}\right)^{1 / 6} .
$$

Then, letting $\log C$ represent the constant terms other than $-\frac{1}{2} \log 2 \pi$,

$$
\begin{aligned}
\log C= & \log \frac{3}{b}+3(p+2) \log \frac{a}{b}-\left(p+\frac{1}{2}\right) \log p-\left(\frac{a}{b}\right)^{3}+p \\
& -\frac{1}{12 p}+\frac{1}{360 p^{3}}-\frac{1}{1260 p^{5}}+\cdots \\
= & \frac{1}{36 p}-\frac{1}{81 p^{2}}+\frac{1}{360 p^{3}}+\frac{2}{1215 p^{4}}-\frac{891}{306180 p^{5}}+\cdots
\end{aligned}
$$

and

$$
\log \frac{f}{C}=-\frac{1}{2} \log 2 \pi-\frac{x^{2}}{2}+\frac{a^{2}}{3}\left[-\frac{1}{4}\left(\frac{x}{a}\right)^{4}+\frac{1}{5}\left(\frac{x}{a}\right)^{5}-\frac{1}{6}\left(\frac{x}{a}\right)^{6}+\cdots\right]
$$

or, limiting the series to the seventh power of $1 / a$,

$$
\begin{aligned}
\frac{f}{C}=\{1 & +\frac{a^{2}}{3}\left[-\frac{1}{4}\left(\frac{x}{a}\right)^{4}+\frac{1}{5}\left(\frac{x}{a}\right)^{5}-\frac{1}{6}\left(\frac{x}{a}\right)^{6}\right. \\
& \left.+\frac{1}{7}\left(\frac{x}{a}\right)^{7}-\frac{1}{8}\left(\frac{x}{a}\right)^{8}+\frac{1}{9}\left(\frac{x}{a}\right)^{9}-\cdots\right] \\
& +\frac{a^{4}}{9}\left[\frac{1}{32}\left(\frac{x}{a}\right)^{8}-\frac{1}{20}\left(\frac{x}{a}\right)^{9}+\frac{37}{600}\left(\frac{x}{a}\right)^{10}-\frac{29}{420}\left(\frac{x}{a}\right)^{11}+\cdots\right] \\
& \left.+\frac{a^{6}}{162}\left[-\frac{1}{64}\left(\frac{x}{a}\right)^{12}+\frac{3}{80}\left(\frac{x}{a}\right)^{13}+\cdots\right]+\cdots\right\} \frac{1}{(2 \pi)^{1 / 2}} \exp \left(-x^{2} / 2\right) .
\end{aligned}
$$

In calculating series (16), the absence of the cubic term as well as the linear term in (15) is appreciated. For calculating percentiles in the range of from 0.005 to $0.995,|x|$ is approximately 2.7 at most, while for $p \geqq 16$, one can see from (12) that $a$ is approximately 12 at least. Some significant figures may be gained in the transformation (5). When $p=100, a \sim 30$, so that two significant figures are gained when $x$ is near zero and one is gained when $x=3$. 
3. Integration. In integrating (16), note that

$$
A_{k}=\int_{0}^{x} t^{2 k+1} \cdot \exp \left(-t^{2} / 2\right) \cdot d t=2^{k} \int_{0}^{x}\left(t^{2} / 2\right)^{k} \cdot \exp \left(-t^{2} / 2\right) \cdot d\left(t^{2} / 2\right)
$$

and

$$
B_{k}=\int_{0}^{x} t^{2 k} \cdot \exp \left(-t^{2} / 2\right) \cdot d t=2^{k-1 / 2} \int_{0}^{x}\left(t^{2} / 2\right)^{k-1 / 2} \cdot \exp \left(-t^{2} / 2\right) \cdot d\left(t^{2} / 2\right) .
$$

The left members of (17) and (18) are called "incomplete normal moment functions" by K. Pearson [2, p. xix], and are tabulated in Table IX of [1], up to $2 k=10$. The $A_{k}$ 's and the $B_{k}$ 's may be evaluated through series (3) or its alternatives.

In terms of the $A_{k}$ 's and the $B_{k}$ 's

$$
\begin{aligned}
I(x)=\int_{0}^{x} f(x) \cdot d x=(C / & \left.(2 \pi)^{1 / 2}\right)\left\{\left[B_{0}-\frac{1}{12 a^{2}} B_{2}-\frac{1}{18 a^{4}} B_{3}\right.\right. \\
+ & \left.\frac{1}{24 a^{4}}\left(\frac{1}{12}-\frac{1}{a^{2}}\right) B_{4}+\frac{37}{5400 a^{6}} B_{5}-\frac{1}{10368 a^{6}} B_{6}\right] \\
+ & {\left[\frac{1}{15 a^{3}} A_{2}+\frac{1}{21 a^{5}} A_{3}-\frac{1}{9 a^{5}}\left(\frac{1}{20}-\frac{1}{3 a^{2}}\right) A_{4}\right.} \\
& \left.\left.-\frac{29}{3780 a} A_{5}+\frac{1}{4320 a^{7}} A_{6}\right]\right\} .
\end{aligned}
$$

Note that $B_{0} /(2 \pi)^{1 / 2}$ corresponds to the normal distribution. It is by far the major contributor to the value of $I(x)$. For convenience $\exp \left(-x^{2} / 2\right)$, which is a factor of every $A_{k}$ and $B_{k}$, may be factored out. Also, it may be useful to note the following relationships:

$$
2(k+1) A_{k}{ }^{\prime}=x^{2 k+2}+A_{k+1}^{\prime},
$$

and

$$
(2 k+1) B_{k}{ }^{\prime}=x^{2 k+1}+B_{k+1}^{\prime},
$$

where the primes indicate that $\exp \left(-x^{2} / 2\right)$ has been factored out.

In integration a constant must be evaluated, and this presents a problem. Note that $x$ is actually measured from the mode of (6), corresponding to the point $y=p+\frac{2}{3}$ in (4). The integral desired starts from $y=0$ (or $\left.y=\infty\right)$. The derivations to this point have been essentially analytic. In order to complete the integration in this spirit, formula (3) may be used for the left tail area. For any given $p$, the point which separates the domains of the two integrals varies according to the accuracy desired; also, the point varies with the $p$. For $p=100, x=-2.5$ may be a good point, but for $p=1000, x=-3$ or -4 may be a better point. If $p$ is high enough, $x$ may be minus six, in which case there is probably no need for the $F_{p}\left(y_{0}\right)$ term. But this leaves the problem of evaluating the $A_{k}$ 's and $B_{k}$ 's to the desired accuracy when $|x|$ is as large as six. This may be accomplished by including a table within the program. Formula (3) converges within a reasonable number of terms if $y / p$ is sufficiently small. The factor that precedes the series may be evaluated from (15), taking a sufficient number of terms. Then, having selected $x_{0}$ 
and the corresponding value $y_{0}$,

$$
F_{p}(y)=F_{p}\left(y_{0}\right)-I\left(x_{0}\right)+I(x)
$$

is the final integral.

Autonetics

3370 Miraloma Avenue

Anaheim, California 92803

1. K. Pearson, Tables for Statisticians and Biometricians, Part I, Cambridge University Press for the Biometrika Trustees, 1914.

2. K. Pearson, Tables of the Incomplete Gamma Function, Cambridge University Press for the Biometrika Trustees, 1957.

3. E. B. Wilson \& Margaret Hilferty, "The distribution of $\chi^{2}$," Proc. Nat. Acad. Sci. v. 17,1931 .

\section{Doppler Broadening Integrals*}

\section{By Van E. Wood, R. P. Kenan and M. L. Glasser}

Asymptotic expansions in terms of Chebyshev polynomials of the integrals

$$
I_{n}(x, v)=v^{1 / 2} \int_{-\infty}^{\infty} y^{n}\left(1+y^{2}\right)^{-1} \exp \left[-v(y-x)^{2}\right] d y, \quad n=0,1, \cdots
$$

have recently been given by Thompson [1]. In this note, we wish to point out that these integrals can be expressed in terms of tabulated (and readily calculable) functions for any values of the parameters, a fact which is not mentioned in the literature we have seen on this subject. Specifically, we find

$$
\begin{aligned}
I_{0}(x, v) & =\pi v^{1 / 2} \operatorname{Re}\left[\exp \left[v(1+i x)^{2}\right] \operatorname{erfc}\left(v^{1 / 2}(1+i x)\right)\right] \\
I_{1}(x, v) & =\pi v^{1 / 2} \operatorname{Im}\left[\exp \left[v(1+i x)^{2}\right] \operatorname{erfc}\left(v^{1 / 2}(1+i x)\right)\right] .
\end{aligned}
$$

These results may be obtained by introducing the representation $\left(1+y^{2}\right)^{-1}=$ $\int_{0}^{\infty} e^{-z} \cos y z d z$, or by solving the coupled differential equations $d I_{0} / d x=$ $2 v\left(I_{1}-x I_{0}\right), d I_{1} / d x=2 v\left(\pi^{1 / 2}-I_{0}-x I_{1}\right)$, or by simply making appropriate changes of variable in some tabulated integrals [2]. Now a method for calculating error functions of complex argument using a rapidly converging infinite series has been described by Salzer [3]; hence the integrals can be obtained easily without using the asymptotic expansion.

By introducing the well-known asymptotic expansion for the co-error function, one may obtain in a very simple way the asymptotic expansions in Chebyshev polynomials

$$
\begin{aligned}
& I_{0} \sim \pi^{1 / 2} \sum_{m=0}^{\infty}(2 m-1) ! !(-2 v)^{m}\left(1+x^{2}\right)^{-m-1 / 2} T_{2 m+1}\left(\left(1+x^{2}\right)^{-1 / 2}\right), \\
& I_{1} \sim \pi^{1 / 2} \sum_{m=0}^{\infty}(2 m-1) ! !(+2 v)^{m}\left(1+x^{2}\right)^{-m-1 / 2} T_{2 m+1}\left(x /\left(1+x^{2}\right)^{1 / 2}\right) .
\end{aligned}
$$

where

Received February 28, 1966.

* Work supported in part by United States Air Force Office of Scientific Research Grant number AF-AFOSR-260-63. 